\title{
Device Alarm System
}

National Cancer Institute

\section{Source}

National Cancer Institute. Device Alarm System. NCI Thesaurus. Code C63033.

Problem associated with the alarm system of the device. 